\title{
Synthesis, Characterization And Antimicrobial Efficacy Of Quinoline Based Compounds
}

\author{
P. J. Vora ${ }^{1}$, A. G. Mehta ${ }^{2}$ \\ ${ }^{1}$ (Department of chemistry, Veer Narmad South Gujarat University, India) \\ ${ }^{2}$ (Department of chemistry, Sir P. T. Sarvajanik College of Science ,India)
}

\begin{abstract}
The required quinoline based compounds IVa-g were prepared by reaction of aryl amine and ethyl acetoacetate via 4-chlorobenzaldehyde(6-methoxy-2-methylequinolin-4-yl)hydrazone(IV) and substituted diazotizedsulfonamides to 4-\{[(4-chlorophenyl)(6-methoxy-2-methylequinolin-4-yl)carbonohydrazonoyl diazenyl\} substituted benzenesulfonamides(IVa-g). The resulting newly synthesized compounds are characterized by elemental analysis, IR, ${ }^{I} H N M R$ and ${ }^{13} C N M R$. All the newly synthesized compounds have been evaluated for their antibacterial activity towards two Gram positive and two Gram negative bacteria and antifungal activity towards Aspergillus niger and Candida albicans. Some selected synthesizes compounds have also been evaluated for their antitubercular activity with mycobacterium tuberculosis bacilli. The results obtained from antimicrobial activity are found that some compounds have higher antibacterial activity and antifungal activity, where as the rest of the compounds show varying activity. Some of the selected compounds show higher antitubercular activity.
\end{abstract}

Keywords - Antibacterial Activity, Antifungal Activity, Antitubercular Activity, Azosulfonamide, Quinoline derivatives, sulfonamide.

\section{INTRODUCTION}

Quinoline derivatives possess various biological activities [1-6]. Some 4-substituted quinoline derivatives showed enhanced activity against gram-negative bacteria [7-8]. Sulfonamides are drugs of proven therapeutic importance [9] and used against a wide spectrum of bacterial ailments [10-13].

Some sulfonamidequinoline derivatives [14] and azobenzenesulfonamide derivatives [15] have been found to be biologically active. One such area in which azo dyes are well known for dyeing to textile materials, while their pharmaceutical activity $[16,17]$ is also known; some are useful as chemotherapeutic agent [18] and some of organic dyes have been used extensively as antibacterial agents [19].

Few compounds of $\mathrm{N}^{1}$ [substituted benzylidine hydrazino] $-\mathrm{N}^{2}$-(substituted quinolinyl)azobenzenes have been found to show antitubercular activity [20-21]. Hence, it was thought interesting to evaluate the antitubercular activity of some selected compounds.

Realizing the medicinal importance of azo compounds [22-26], quinoline derivatives and sulfonamides, it was considered worthwhile to incorporate these two moieties. It was therefore thought interesting to synthesize the title compounds with an object of ascertaining whether such compounds could augment their antibacterial and antifungal activity.

\subsection{Materials}

\section{EXPERIMENTAL}

All reagents were obtained from commercial sources. Solvents were dried and purified with known conventional methods.

\subsection{Analytical Method}

All melting points were taken in open capillary tubes and were uncorrected. The IR spectra were obtained on a Perkin-Elmer BX series FT-IR-5000 spectrophotometer using $\mathrm{KBr}$ pellets. The ${ }^{1} \mathrm{H}$ NMR and ${ }^{13} \mathrm{C}$ NMR spectra in DMSO- $\mathrm{d}_{6}$ and $\mathrm{CDCl}_{3}$ were recorded on Varian Gemini $400 \mathrm{MHz}$ spectrometer and chemical shifts were reported as parts per million $(\delta \mathrm{ppm})$ downfield using TMS as an internal standard.

\subsection{Preparation of 2-methyl-6-methoxy-4-chloroquinoline}

\subsubsection{Ethyl- $\beta$-4-methoxyanilinocrotonate}

A mixture of p-anisidine $(0.05 \mathrm{~mol})$ and acetoacetic ester $(0.05 \mathrm{~mol})$ with a trace of concentrated hydrochloric acid was kept in a desiccator for $24 \mathrm{hr}$. The residue was cyclized by PPA.

2.3.2 Polyphosphoric acid (PPA) 
Polyphosphoric acid was prepared by dissolving phosphorus pentoxide (40.0 g) into orthophosphoric acid (24 $\mathrm{ml} ; \delta=1.75$ ). The mixture was heated at $95-100^{\circ} \mathrm{C}$ for half an hour; the scum was removed and clear solution thus obtained was used for the cyclization step.

\subsubsection{2-methyl-6-methoxy-4-hydroxyquinoline(I)}

The crude crotonate was mixed with freshly prepared PPA at room temperature, stirred well for some time and then the temperature was raised to $100^{\circ} \mathrm{C}$ effervescences and was kept in desiccators for $24 \mathrm{hr}$. Next day, the temperature was slowly raised and lowered by $10^{\circ} \mathrm{C}$ until it reached $140^{\circ} \mathrm{C}$ over $1 \mathrm{hr}$. This treatment helps in getting clean product in high yield. The reaction mass was cooled and decomposed with crushed ice and neutralized with liquor ammonia on the acidic side. The product was filtered washed with water dried and crystallized from alcohol. Yield 63\%; mp: $300^{\circ} \mathrm{C}$; Anal. Calcd for $\mathrm{C}_{11} \mathrm{H}_{11} \mathrm{O}_{2} \mathrm{~N}(189.0)$ : C, 69.84; H, 5.82; N, 7.40. Found: C, 69.76; H, 5.76; N, 7.35.

\subsubsection{2-methyl-6-methoxy-4-chloroquinoline(II)}

2-methyl-6-methoxy-4-hydroxyquinoline $(3.0 \mathrm{~g})$ was refluxed with phosphorus oxychloride $(25.0 \mathrm{ml})$ for one hour. After cooling to room temperature, it was poured in ice and neutralized with liquor ammonia on the acidic side, when a voluminous mass of chloro compound separated. The product was washed with water and crystallized from ethanol. Yield $74 \%, \mathrm{mp} 92{ }^{\circ} \mathrm{C}$. Anal. Calcd for $\mathrm{C}_{11} \mathrm{H}_{10} \mathrm{NOCl}$ (207.5): C, 63.61; H, 4.81; N, 6.75. Found: C, 63.53; H, 4.73; N, 6.70 .

\subsection{Preparation of 2-methyl-6-methoxy-4-quinolinylhydrazine(III)}

A mixture of 2-methy-6-methoxy-4-chloroquinoline $(4.24 \mathrm{gm}, 0.02 \mathrm{M})$ and hydrazine hydrate $(5.0 \mathrm{ml}$, $0.02 \mathrm{M}$ ) was refluxed for 5 hours in absolute alcohol in a water bath. The reaction mixture was transferred in an evaporating dish and allowed to solidify. It was then treated with water and filtered. The resulting product was crystallized from alcohol.Yield 76\%; mp: $282^{\circ} \mathrm{C}$. Anal. Calcd for $\mathrm{C}_{11} \mathrm{H}_{13} \mathrm{~N}_{3} \mathrm{O}$ (203.0): C, 65.02; H, 6.41; N, 20.68. Found: C, 64.95; H, 6.31; N, 20.60.

\subsection{Preparation of 4-chlorobenzaldehyde (6-methoxy-2-methylequinolin-4-yl) hydrazone(IV)}

A mixture of 6-methoxy-4-hydrazino-2-methylquinoline $(1.0 \mathrm{gm}, 0.005 \mathrm{M})$ and 4-aminobenzaldehyde $(0.75 \mathrm{gm}, 0.005 \mathrm{M})$ was refluxed for 2 hours in glacial acetic acid at $80^{\circ} \mathrm{C}$ in water bath. The reaction mixture was cooled, poured in ice water and neutralized with liquor ammonia in slightly acidic side. The resulting product was crystallized from alcohol.

Yield 75\%; mp: 210 ${ }^{\circ} \mathrm{C}$; FT-IR [v, $\left.\mathrm{cm}^{-1}, \mathrm{KBr}\right]: 3325(\mathrm{~N}-\mathrm{H}), 1369$ (C-N), 1255 (C-O-C), 2943(C-H, aromatic), $1610(\mathrm{C}=\mathrm{C}), 1313\left(\mathrm{Ar}-\mathrm{CH}_{3}\right), 765(\mathrm{C}-\mathrm{Cl}) .{ }^{1} \mathrm{H}$ NMR $\left[400 \mathrm{MHz}, \delta, \mathrm{ppm}, \mathrm{DMSO}-\mathrm{d}_{6}\right]: 7.89(1 \mathrm{H},-\mathrm{NH}), 2.57(3 \mathrm{H},-$ $\left.\mathrm{CH}_{3}\right), 3.96\left(3 \mathrm{H},-\mathrm{OCH}_{3}\right), 7.45-7.8(8 \mathrm{H}, \mathrm{Ar}-\mathrm{H}), 8.6(1 \mathrm{H},-\mathrm{CH}=\mathrm{N}) .{ }^{13} \mathrm{C}$ NMR [400 MHz, $\delta$, ppm, DMSO-d $\left.\mathrm{d}_{6}\right]: 21(-$ $\left.\mathrm{CH}_{3}\right), 129$ (Benzene), 160(Quinoline), 56((-OCH$)_{3}$ ). Anal. Calcd for $\mathrm{C}_{18} \mathrm{H}_{16} \mathrm{~N}_{3} \mathrm{OCl}(325.5): \mathrm{C}, 66.35 ; \mathrm{H}, 4.91 ; \mathrm{N}$, 12.90. Found: C, 66.19; H, 4.75; N, 12.35 .

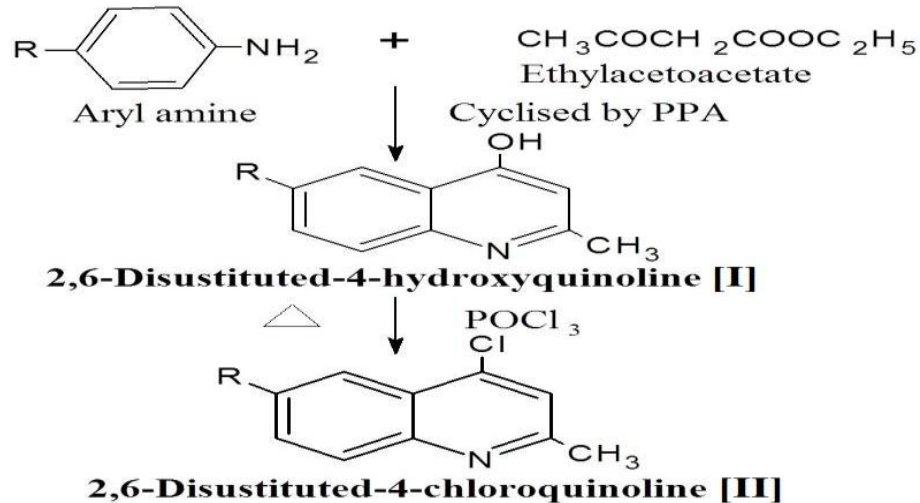




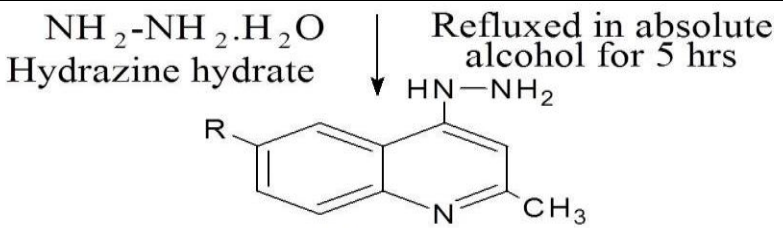

2,6-Disustituted-4-quinolinyl hydrazine [III]
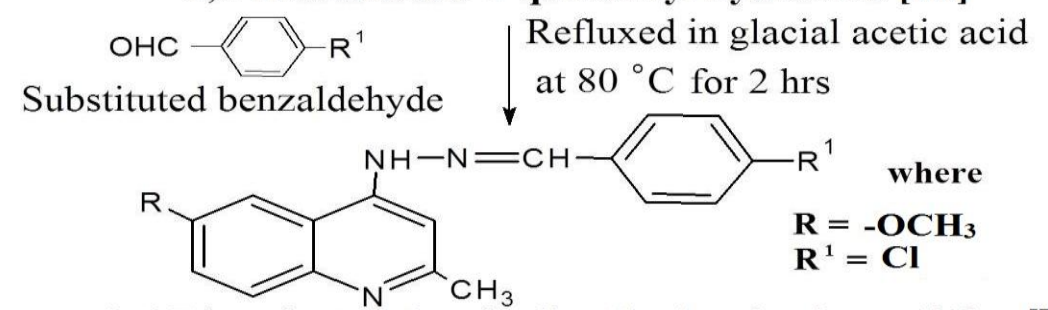

2,6-Disustituted-4-quinolinyl hydrazino benzylidine [IV]

Figure 1 Synthesis of 4-chlorobenzaldehyde(6-methoxy-2-methylequinolin-4-yl) hydrazone(IV)

In Fig. $2 \mathbf{R}^{2}=-\mathrm{H}$,

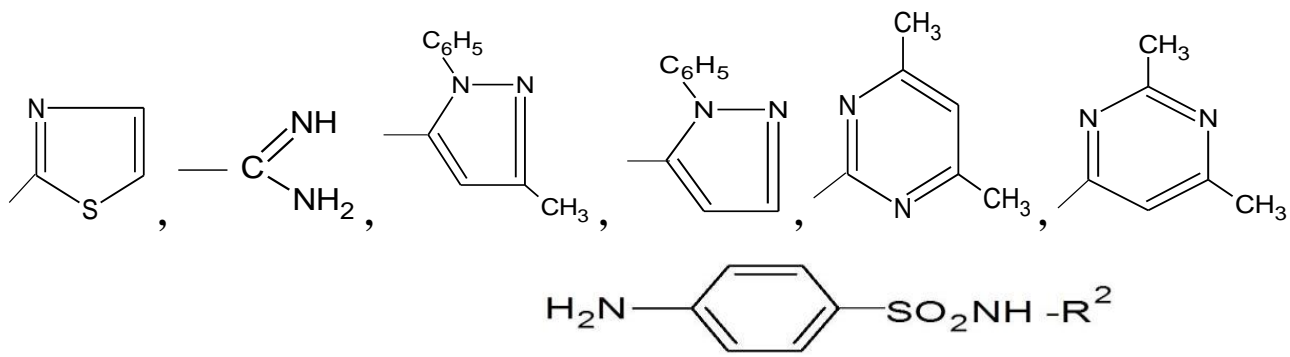

Substituted sulfonamides

$\mathrm{O}-5^{\circ} \mathrm{C} \downarrow$ Diazotisation

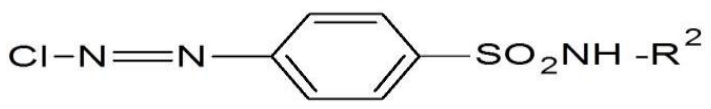

Substituted diazotised sulfonamides $[\mathrm{E}]$

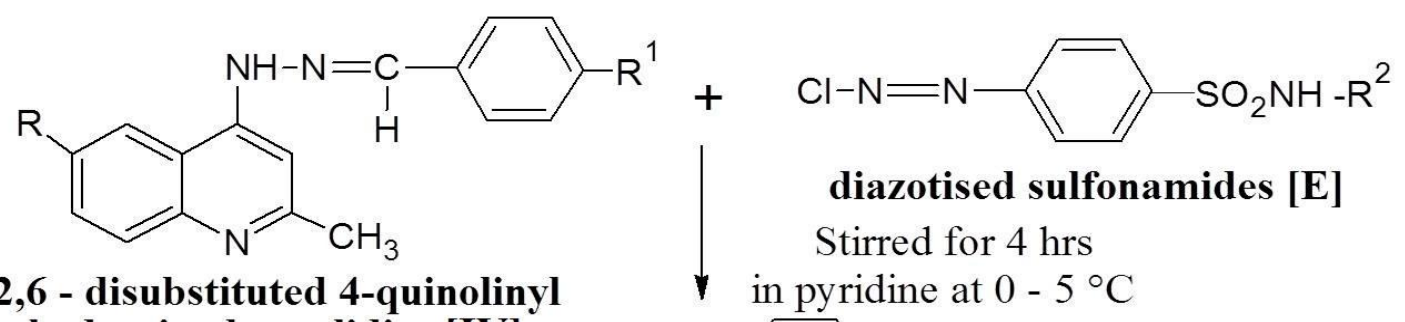
hydrazino benzylidine [IV]

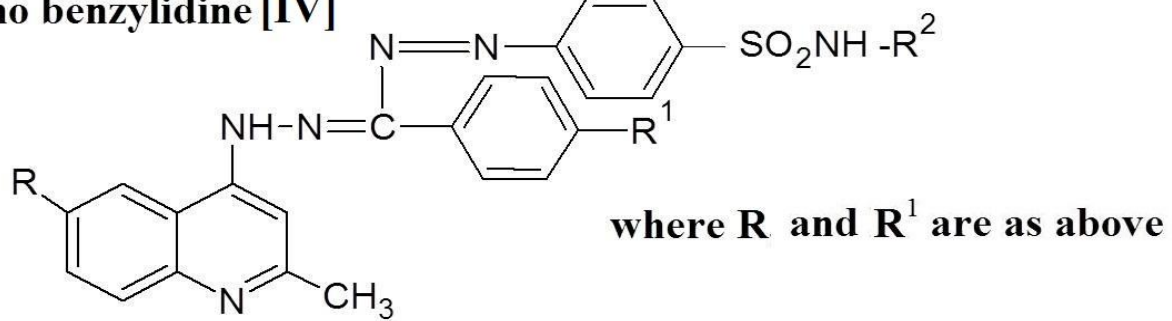

substituted - $\left[\left(4^{\prime}\right.\right.$-substituted benzylidine $)$ hydrazino

$-\mathrm{N}^{2}-2,6$-disubstituted quinolinyl] azosulfonamides

Figure 2 Synthesis route of compounds Iva-g 
2.6 General procedure for the preparation of 4-\{[(4-chlorophenyl)(6-methoxy-2-methylequinolin-4yl)carbonohydrazonoyl]diazenyl\}substituted benzenesulfonamides (IVa-g)

2.6.1 IVa:4-\{[(4-chlorophenyl)(6-methoxy-2-methylequinolin-4-yl)carbonohydrazonoyl]diazenyl $\}$

benzenesulfonamides

\subsubsection{Diazotisation of sulfanilamide:}

Sulfanilamide $(0.774 \mathrm{gm}, 0.0045 \mathrm{M})$ was dissolved in hydrochloric acid $(10 \mathrm{ml}, 50 \%)$ and the solution was cooled to $0-5^{\circ} \mathrm{C}$. A solution of sodium nitrite $(0.5 \mathrm{gm}, 0.0045 \mathrm{M})$ in water $(2 \mathrm{ml})$ previously cooled to $0^{\circ} \mathrm{C}$ was then added over a period of five minutes with constant stirring and maintaining the temperature of the mixture at $0-5^{\circ} \mathrm{C}$; stirring was continued for half an hour, maintaining the same temperature with positive test for nitrous acid on starch iodide paper. Excess of nitrous acid was destroyed by adding the required quantity of sulfamic acid. The resulting solution was used for coupling reaction.

Following the above procedure, other sulfonamides were diazotized and used for coupling reaction.

\subsubsection{Coupling of diazotized solution with IVa:}

A clear solution of IV $(1.52 \mathrm{gm} ; 0.0045 \mathrm{M})$ in pyridine $(5 \mathrm{ml})$ was cooled below $5^{\circ} \mathrm{C}$. To this well stirred solution, diazotized solution was added dropwise over a period of 10-15 minutes, maintaining the $\mathrm{pH} 7$ to 7.5 . The stirring was continued for 4 hours at $0-5^{\circ} \mathrm{C}$. The product was filtered, washed with water and crystallized from glacial acetic acid.

Yield 70.0\%; mp: $220^{\circ} \mathrm{C}$; FT-IR [v, $\left.\mathrm{cm}^{-1}, \mathrm{KBr}\right]: 3327(\mathrm{~N}-\mathrm{H}), 1327\left(\mathrm{Ar}^{\left.-\mathrm{CH}_{3}\right)}, 1371(\mathrm{C}-\mathrm{N}), 688(\mathrm{C}-\mathrm{S})\right.$, $1568(\mathrm{~N}=\mathrm{N}), 1186\left(\mathrm{~S}=\mathrm{O}, \mathrm{RSO}_{2} \mathrm{NH}_{2}\right), 1055(\mathrm{C}-\mathrm{O}-\mathrm{C}),{ }^{1} \mathrm{H}$ NMR [400 MHz, $\delta, \mathrm{ppm}, \mathrm{DMSO}-\mathrm{d}_{6}$ ]: 7.0-7.93 (8H, Ar$\mathrm{H}), 2.55\left(3 \mathrm{H},-\mathrm{CH}_{3}\right), 3.40\left(3 \mathrm{H},-\mathrm{OCH}_{3}\right), 8.85(1 \mathrm{H},-\mathrm{NH})$. Anal. Calcd for $\mathrm{C}_{24} \mathrm{H}_{21} \mathrm{~N}_{6} \mathrm{O}_{3} \mathrm{SCl}(508.5): \mathrm{C}, 56.63 ; \mathrm{H}$, 4.12; N, 16.51. Found: C, 56.57; H, 4.02; N, 16.45 .

2.6.2 4-\{[(4-chlorophenyl)(6-methoxy-2-methylquinolin-4-yl)carbonohydrazonoyl] diazenyl\}-N-1,3-

thiazol-5-ylbenzenesulfonamide (IVb)

Yield 73\%; mp: $305^{\circ} \mathrm{C}$; FT-IR [v, cm $\left.{ }^{-1}, \mathrm{KBr}\right]: 3335(\mathrm{~N}-\mathrm{H}), 1319\left(\mathrm{Ar}^{-\mathrm{CH}_{3}}\right), 1271(\mathrm{C}-\mathrm{N}), 1029(\mathrm{C}-\mathrm{O}-$ C). $1595(\mathrm{~N}=\mathrm{N}), 1186\left(\mathrm{~S}=\mathrm{O}, \mathrm{RSO}_{2} \mathrm{NH}_{2}\right), 624(\mathrm{C}-\mathrm{S}),{ }^{1} \mathrm{H}$ NMR $\left[400 \mathrm{MHz}, \delta, \mathrm{ppm}, \mathrm{DMSO}-\mathrm{d}_{6}\right]: 2.55\left(3 \mathrm{H},-\mathrm{CH}_{3}\right)$, 8.85(1H,-NH), 7.0-7.93 $(14 \mathrm{H}, \mathrm{Ar}-\mathrm{H}), 7.38\left(1 \mathrm{H},-\mathrm{SO}_{2} \mathrm{NH}\right), 3.40\left(3 \mathrm{H},-\mathrm{OCH}_{3}\right) .{ }^{13} \mathrm{C}$ NMR [400 MHz, $\delta, \mathrm{ppm}$, $\mathrm{CDCl}_{3}$ ]: 21(- $\left.\mathrm{CH}_{3}\right)$, 119(thiazole), 129(benzene), 154(quinoline), 160(imine), 56(-OCH 3 ). Anal.Calcd for $\mathrm{C}_{27} \mathrm{H}_{22} \mathrm{~N}_{7} \mathrm{O}_{3} \mathrm{~S}_{2} \mathrm{Cl}$ (591.5), C, 54.77; H, 3.71; N, 16.56. Found: C, 54.70; H, 3.64; N, 16.39.

2.6.3 N-[amino(imino)methyl]-4-\{[(4-chlorophenyl)(6-methoxy-2-methylquinolin-4-yl) carbonohydrazonoyl] diazenyl $\}$ benzenesulfonamide (IVc)

Yield 72\%; mp: $278^{\circ} \mathrm{C}$; FT-IR [v, $\left.\mathrm{cm}^{-1}, \mathrm{KBr}\right]: 3332(\mathrm{~N}-\mathrm{H}), 1326\left(\mathrm{Ar}_{-} \mathrm{CH}_{3}\right), 1315(\mathrm{C}-\mathrm{N}), 1264(\mathrm{C}-\mathrm{O}-$ C), $1590(\mathrm{~N}=\mathrm{N}), 1186\left(\mathrm{~S}=\mathrm{O}, \mathrm{RSO}_{2} \mathrm{NH}_{2}\right),{ }^{1} \mathrm{H}$ NMR [400 MHz, $\left.\delta, \mathrm{ppm}, \mathrm{DMSO}-\mathrm{d}_{6}\right]: 2.52\left(3 \mathrm{H},-\mathrm{CH}_{3}\right), 8.85(1 \mathrm{H},-$ $\mathrm{NH})$, 7.0-7.95 (12H, Ar-H), $3.40\left(3 \mathrm{H},-\mathrm{OCH}_{3}\right)$. Anal. Calcd for $\mathrm{C}_{25} \mathrm{H}_{23} \mathrm{~N}_{8} \mathrm{O}_{3} \mathrm{SCl}$ (550.5): C, 54.49; H, 4.17; N, 20.24. Found: C, 54.32; H, 4.11; N, 20.15.

2.6.4 4-\{[(4-chlorophenyl)(6-methoxy-2-methylquinolin-4-yl)carbonohydrazonoyl] diazenyl\}-N-(3-methyl1-phenyl-1H-pyrazol-5-yl)benzenesulfonamide (IVd)

Yield 79\%; mp: 246 ${ }^{\circ}$; FT-IR [v, $\left.\mathrm{cm}^{-1}, \mathrm{KBr}\right]: 3337(\mathrm{~N}-\mathrm{H}), 1324\left(\mathrm{Ar}^{-\mathrm{CH}_{3}}\right), 1282(\mathrm{C}-\mathrm{N}), 1239(\mathrm{C}-\mathrm{O}-$ C), $1568(\mathrm{~N}=\mathrm{N}), 1186\left(\mathrm{~S}=\mathrm{O}, \mathrm{RSO}_{2} \mathrm{NH}_{2}\right),{ }^{1} \mathrm{H}$ NMR [400 MHz, $\delta$, ppm, DMSO-d 6 ]: $2.58\left(3 \mathrm{H},-\mathrm{CH}_{3}\right), 8.82(1 \mathrm{H},-$ NH), 7.0-7.85 (18H, Ar-H). Anal. Calcd for $\mathrm{C}_{34} \mathrm{H}_{29} \mathrm{~N}_{8} \mathrm{O}_{3} \mathrm{SCl}$ (664.5): C, 61.39; H, 4.36; N, 16.85. Found: C, 61.27; H, 4.21; N, 16.66 .

2.6.5 4-\{[(4-chlorophenyl)(6-methoxy-2-methylquinolin-4-yl)carbonohydrazonoyl] diazenyl\}-N-(1-phenyl1H-pyrazol-5-yl)benzenesulfonamide (IVe)

Yield 76\%; mp: $217^{\circ} \mathrm{C}$; FT-IR [v, $\left.\mathrm{cm}^{-1}, \mathrm{KBr}\right]:$ 1324( $\mathrm{Ar}^{\left.-\mathrm{CH}_{3}\right)}, 1580(\mathrm{~N}=\mathrm{N}), 1362(\mathrm{C}-\mathrm{N}), 687(\mathrm{C}-\mathrm{S})$, 1372( $\left.\mathrm{S}=\mathrm{O}, \mathrm{RSO}_{2} \mathrm{NH}_{2}\right) .{ }^{1} \mathrm{H}$ NMR [400 MHz, $\left.\delta, \mathrm{ppm}, \mathrm{DMSO}-\mathrm{d}_{6}\right]: 2.56\left(3 \mathrm{H},-\mathrm{CH}_{3}\right), 8.86(1 \mathrm{H},-\mathrm{NH}), 7.50-7.89$ $(19 \mathrm{H}, \mathrm{Ar}-\mathrm{H}), 3.27\left(3 \mathrm{H},-\mathrm{OCH}_{3}\right) .{ }^{13} \mathrm{C}$ NMR [400 MHz, $\left.\delta, \mathrm{ppm}, \mathrm{CDCl}_{3}\right]$ : 162 (imine), 156(quinoline), $21\left(-\mathrm{CH}_{3}\right)$. Anal. Calcd for $\mathrm{C}_{33} \mathrm{H}_{27} \mathrm{~N}_{8} \mathrm{O}_{3} \mathrm{SCl}(650.5)$ : C, 60.87; H, 4.15; N, 17.21. Found: C, 60.77; H, 4.08; N, 17.05.

2.6.6. 4-\{[(4-chlorophenyl)(6-methoxy-2-methylquinolin-4-yl)carbonohydrazonoyl] diazenyl\}-N-(4,6dimethylpyrimidin-2-yl)benzenesulfonamide (IVf)

Yield 65\%; mp: 215 C; FT-IR [v, $\left.\mathrm{cm}^{-1}, \mathrm{KBr}\right]: 3309(\mathrm{~N}-\mathrm{H}), 1329\left(\mathrm{Ar}^{-} \mathrm{CH}_{3}\right), 1307(\mathrm{C}-\mathrm{N}), 1025(\mathrm{C}-\mathrm{O}-$ C), $1609(\mathrm{~N}=\mathrm{N}), 1176\left(\mathrm{~S}=\mathrm{O}, \mathrm{RSO}_{2} \mathrm{NH}_{2}\right)$. Anal. Calcd for $\mathrm{C}_{30} \mathrm{H}_{27} \mathrm{~N}_{8} \mathrm{O}_{3} \mathrm{SCl}$ (614.5): C, 58.58; H, 4.39; N, 18.22. Found: C, 58.44; H, 4.23; N, 18.10 .

2.6.7 4-\{[(4-chlorophenyl)(6-methoxy-2-methylquinolin-4-yl)carbonohydrazonoyl] diazenyl\}-N-(2,6dimethylpyrimidin-4-yl)benzenesulfonamide (IVg)

Yield 56\%; mp: 235 ${ }^{\circ}$; FT-IR [v, $\left.\mathrm{cm}^{-1}, \mathrm{KBr}\right]: 3317(\mathrm{~N}-\mathrm{H}), 1322\left(\mathrm{Ar}^{-\mathrm{CH}_{3}}\right), 1361(\mathrm{C}-\mathrm{N}), 1273(\mathrm{C}-\mathrm{O}-$ C), $1560(\mathrm{~N}=\mathrm{N}), 1177\left(\mathrm{~S}=\mathrm{O}, \mathrm{RSO}_{2} \mathrm{NH}_{2}\right)$. Anal. Calcd for $\mathrm{C}_{30} \mathrm{H}_{27} \mathrm{~N}_{8} \mathrm{O}_{3} \mathrm{SCl}$ (614.5): C, 58.58; H, 4.39; N, 18.22 . Found: C, 58.44; H, 4.23; N, 18.10 . 


\subsection{Chemistry}

\section{III. . Result \& Discussion}

The final Structures of compounds IVa-g were confirmed on the basis of Infrared spectroscopy and NMR spectroscopy. The IR spectra of compounds shows characteristic bands at $1618-1480 \mathrm{~cm}^{-1}$ (Substituted quinolines), $1330 \mathrm{~cm}^{-1}$ (C-H stretching), $1340-1315 \mathrm{~cm}^{-1}$ (C-N stretching- tertiary amine), $1350-1280 \mathrm{~cm}^{-1}$ (C-N stretching-secondary amine), $1630-1575 \mathrm{~cm}^{-1}\left(\mathrm{~N}=\mathrm{N}\right.$ stretching), $1370-1330 \mathrm{~cm}^{-1}$ and $1180-1160 \mathrm{~cm}^{-}$ ${ }^{1}\left(\mathrm{RSO}_{2} \mathrm{NH}_{2}\right), 779-651 \mathrm{~cm}-1$ (C-S stretching). ${ }^{1} \mathrm{HNMR}$ signal at $82.58\left(-\mathrm{CH}_{3}\right), \delta 7.9(-\mathrm{NH}), 86.9-7.9(\mathrm{Ar}-\mathrm{H}), \delta 8.57(-$ $\mathrm{CH}=\mathrm{N}), \delta 7.41\left(-\mathrm{SO}_{2} \mathrm{NH}\right) .{ }^{13} \mathrm{C}$ NMR signal at $\delta 151-156$ (quinoline), $\delta 43\left(-\mathrm{N}\left(\mathrm{CH}_{3}\right)_{2}\right), \delta 119$ (thaizole).

\subsection{Biological Activity}

\subsubsection{Anti Bacterial Activity}

Antibacterial activities of all the compounds were studied against Gram-positive bacteria [Staphylococcus aureus and Bacillus subtilis] and Gram-negative bacteria [Escherichia coli and Pseudomonas aeruginosa] at a concentration of $100 \mu \mathrm{g} / \mathrm{ml}$ by agar cup plate method. The area of inhibition of zone measured in millimeter. An examination of the data reveals that all compounds showed antibacterial activity. Results are presented in Table 1.

\subsubsection{Anti Fungal Activity}

The synthesized compounds were also screened for their antifungal activity against Candida albicans and Aspergillus niger using the agar cup plate diffusion method by dissolving in DMF at a concentration of 100 $\mu \mathrm{g} / \mathrm{mL}$. The zone of inhibition was measured after 3 days at $20^{\circ} \mathrm{C}$. Results are presented in Table 1.

\subsubsection{Anti Tubercular Activity}

In the present work some selected compounds were also tested for their antitubercular activity against INH sensitive strain of $\mathrm{H}_{37} \mathrm{Rv}$ at $12.5 \mu \mathrm{g} / \mathrm{ml}$ with the help of BacT/ALERT 3D Detection System. Result is presented in Table 1.

Table 1 Anti Microbial activity of 4-\{[(4-chlorophenyl)(6-methoxy-2-methylequinolin-4-yl) carbonohydrazonoyl]diazenyl $\}$ substituted benzenesulfonamides(IVa-g)

\begin{tabular}{|c|c|c|c|c|c|c|c|}
\hline \multirow{3}{*}{ Compound } & \multicolumn{6}{|c|}{ Zone of inhibition (mm) } & \multirow{3}{*}{$\begin{array}{c}\text { Anti Tubercular } \\
\text { Activity } \\
\mu \mathrm{g} / \mathrm{ml}\end{array}$} \\
\hline & \multicolumn{4}{|c|}{ Anti Bacterial Activity } & \multicolumn{2}{|c|}{$\begin{array}{c}\text { Anti Fungal } \\
\text { Activity }\end{array}$} & \\
\hline & E.coli & $\begin{array}{c}\text { Ps. } \\
\text { Aeruginosa }\end{array}$ & $\begin{array}{c}\text { B. } \\
\text { subtilis }\end{array}$ & $\begin{array}{c}\text { S. } \\
\text { aureus }\end{array}$ & $\begin{array}{c}\text { A. } \\
\text { niger }\end{array}$ & $\begin{array}{c}\text { C. } \\
\text { albicans }\end{array}$ & \\
\hline IVa & 08 & 07 & 09 & 10 & 12 & 10 & - \\
\hline IVb & 09 & 09 & 08 & 10 & 10 & 09 & - \\
\hline IVc & 11 & 12 & 16 & 11 & 09 & 08 & - \\
\hline IVd & 09 & 10 & 09 & 09 & 07 & 07 & - \\
\hline IVe & 08 & 09 & 08 & 10 & 08 & 07 & - \\
\hline IVf & 11 & 12 & 10 & 13 & 08 & 08 & Sensitive \\
\hline IVg & 10 & 09 & 08 & 10 & 12 & 10 & - \\
\hline IV & 15 & 14 & 16 & 11 & 9 & 8 & - \\
\hline
\end{tabular}

IV.

. Conclusion

It would be seen from Table-1 that the compounds IVc and IVf exhibit highest antibacterial activity against both Gram negative and Gram Positive organisms. Comparing the antibacterial activity of these compounds with the parent compound IV, it is surprising to note that all the compounds show decrease in the antibacterial activity against Gram negative bacteria. In most of the cases the antibacterial activity against Gram positive bacteria is either decreased or retained. Table-1 indicates that the highest antifungal activity is exhibited by the compounds IVa against both the fungi. The rest of the compounds do not show any appreciable antifungal activity against both fungi. Comparing the antifungal activity of these products with the parent compounds it is seen that the compound IVa and IVg show appreciable additive antifungal activity against A.niger and C.albicans. In rest of these compounds either the antifungal activity is decreased or retained against both the fungi. It is surprising to 
note that compound IVf having 6-methoxy group in the quinoline ring with sulfadimidine as diazo components show sensitive respo nse at $12.5 \mu \mathrm{g} / \mathrm{ml}$ against INH sensitive strain of $\mathrm{H}_{37} \mathrm{Rv}$.

\section{Acknowledgements}

I express my sincere thanks to Dr. A. G. Mehta, Principal, Sir P.T. Sarvajanik College of Science for providing me guidance and necessary research facilities to carry out this work at the college laboratory. I also thanks Miss S. Aparna, IAS, Commissioner, SMC for giving me permission to do anti-tubercular activity at the Microbiology Department, SMIMER, Surat.

\section{REFERENCES}

[1] Lednicer and L. A. Mitscher, Org. Chem. Drug Synth., 1, 341 (1975).

[2] J. H. Burckhalter, W. T. Brinigar and P. E. Thomson, J. Org. Chem., 26, 4070 (1961).

[3] D. Prakash, S. Kumar and S. M. Prasad, J. Ind. Chem. Soc., 65, 771 (1988).

[4] R. Rodriguez, Ger. Pat., 2066638 (1970); Chem. Abstr., 73, 9879 (1970).

[5] F. F. Ehetino and G. C. Wright, Fr. Pat., 1388756 (1965).

[6] P. H. Desai and K. R. Desai, J. Ind. Chem. Soc., 65, 805 (1988)

[7] V. S. Misra and V. K. Saxena, J. Prakt. Chem., 5, 314, 958 (1972).

[8] S. E. Hawkins and J. M. Hainton, Microbiol., 5, 57 (1972).

[9] Surendra Bahadur and Mukta Saxena, J. Ind. Chem. Soc., 60, 684 (1983).

[10] D. B. Clavson, J. A. S. Pringle and G. M. Ranses, Biochem. Pharmacol., 16, 614 (1967).

[11] W. V. D. Bassche, Pharm. Tijdsehm Belg., 42, 156 (1965)

[12] W. N. Beerley, W. Peters and K. Mager, Ann. Trop. Med. Parasitol., 62, 288 (1960).

[13] G. Tarbini, Inst. Congr. Chemother. Proc., $5^{\text {th }}, 2(2), 909$ (1967).

[14] A. R. Shah, C. M. Desai and B. M. Desai, J. South Guj. Uni., Surat, 7, 85 (1978); J. Inst. Chemists (India), 59,257 (1987).

[15] A. R. Shah, C. M. Desai and B. M. Desai, J. Inst. Chemists (India), 60, 15 (1988).

[16] /K. N. Gaind and J. M. Khanna, Ind. J. Pharm., 26, 34 (1964).

[17] K. N. Gaind and S. K. Gulati, Ind. J. Pharm., 28, 272 (1966).

[18] L. S. Goodman and A. Gilman, "The Pharmacological basis of Therapeutics", $4^{\text {th }}$ Ed., Mac Millan, New York, P.III (1970).

[19] Anjani Solankee, Ph. D. Thesis, South Guj. Uni., Surat, 234 (1984).

[20] D. C. Tandel, Ph.D. Thesis, South Gujarat Uni., Surat, 196(1993).

[21] Jigna K. Machhi, Ph.D. Thesis, South Gujarat Uni., Surat, 205(2000).

[22] Burger, “A Medicinal Chemistry”, Vol. 1, p.668, John Willey, New York (1970).

[23] A. Goerner and H. L. Haley, J. Tab. Clin. Med., 16, 957 (1931).

[24] C. O. Wilson Ole Gisvold, Robert F. Doerge and B. Lippincott, "A Text Book of Organic Medicinal Pharmaceutical Chemistry”, $6^{\text {th }}$ Ed., 193 (1971).

[25] L. S. Goodman and A. Gilman, "The Pharmacological basis of Therapeutics”, p. 1009, $5^{\text {th }}$ Ed., Mac Millan Publishing Company, Inc., New York, (1975).

[26] J. Bhagwan, Y. C. Joshi, R. P. Tyagi, B. C. Joshi and H. N. Mangal, J. Inst. Chemists (India), 55, 58 (1983). 